\title{
Application of the maximum entropy method in texture analysis
}

\author{
Thomas Böhlke
}

Otto-von-Guericke-Universität Magdeburg, Institut für Mechanik, PSF 4120, D-39016 Magdeburg, Germany

\begin{abstract}
The problem of estimating the crystallite orientation distribution function (codf) based on the leading texture coefficients is considered. Problems of such a type are called moment problems, which are well known in statistical mechanics and other areas of science. It is shown how the maximum entropy method can be applied to estimate the codf. Special emphasis is given to a coordinate-free formulation of the problem. The codf is represented by a tensorial Fourier series. The equations, which have to be solved for the estimate of the distribution function, are derived for all tensor ranks of the Fourier coefficients. As a numerical example, a model codf is estimated based on a set of discrete crystal orientations given by a full-constrained Taylor type texture simulation.
\end{abstract}

(c) 2004 Elsevier B.V. All rights reserved.

Keywords: Crystallite orientation distribution function; Crystallographic texture; Maximum entropy method; Tensorial Fourier expansion

\section{Introduction}

The distribution of crystal orientations is an important microstructural feature which affects the overall properties of polycrystalline metals. If this distribution is inhomogeneous, the material has a crystallographic texture. Such a texture influences the overall elastic and the overall viscoplastic

E-mail address: boehlke@mb.uni-magdeburg.de behavior as well as the non-mechanical properties of a polycrystal.

The distribution of crystal orientations can be described by correlation functions. In texture analysis, the one-point correlation function is called crystallite orientation distribution function (codf). It specifies the volume fraction of crystals having a specific orientation. A crystal orientation can be identified by a proper orthogonal tensor.

There exist different approaches to the representation of the codf. The classical representation by generalized harmonic functions was introduced 
by Bunge [7] and Roe [13]. Later on, Adams et al. [1] and Guidi et al. [9] introduced a tensorial Fourier expansion of the codf (see also [21,22]). Both representations are generally equivalent. The advantage of the tensorial representation is that it is coordinate-free. Therefore, the texture coefficients that occur in the tensorial representation can be used as micro-mechanically defined and measurable internal variables in continuum mechanics [4-6].

In the present paper the following problem is considered: how to estimate the codf, if a fixed number of discrete crystal orientations or leading texture coefficients is known? These orientation data can be obtained experimentally for example by automated electron back scatter diffraction orientation measurements. The estimate of the codf has to be consistent in the way that it is nonnegative and normalized. Such a type of a problem is usually called moment problem. Moment problems are well known in statistical mechanics and other areas of science. The estimate of a distribution function based on incomplete data is not unique. One way to single out a solution of the aforementioned problem is to maximize the (information-theoretic) entropy of the codf. The maximum entropy method was introduced by $[10,11]$ into the field of statistical mechanics. For an overview on this method see $\mathrm{Wu}$ [20].

The outline of the paper is as follows: in Section 2 the basic formula of the tensorial representation of the codf is summarized for the special case of a cubic crystal symmetry. In Section 3 the moment problem is stated in terms of tensorial texture coefficients. Then it is shown that the so called crystallographic exponential family, here introduced with tensorial coefficients, solves the moment problem. As a numerical example a model codf is estimated based on a set of discrete crystal orientations given by a full-constrained Taylor type texture simulation.

Notation. Fourth- and higher-order tensors are denoted by, e.g., $\mathbb{V}_{\langle\alpha\rangle}$, where the index $\alpha$ specifies the tensor rank. The corresponding zero tensor is written as $\mathbb{O}$. The dyadic product and the Frobenius norm are denoted by $\otimes$ and $\|\cdot\|$, respectively. Components of tensors are always represented with respect to an orthonormal vector basis $\boldsymbol{e}_{i}$ in the three-dimensional Euclidean space. For exam- ple, a fourth-order tensor is written in the following way: $\mathbb{V}_{\langle 4\rangle}=V_{i j k l} \boldsymbol{e}_{i} \otimes \boldsymbol{e}_{j} \otimes \boldsymbol{e}_{k} \otimes \boldsymbol{e}_{l}$. The scalar product between tensors is denoted by a dot, e.g., $\mathbb{V}_{\langle\alpha\rangle} \cdot \mathbb{F}_{\langle\alpha\rangle}$. A linear mapping is written as $\mathbb{V}_{\langle\alpha\rangle}=\mathbb{E}_{\langle 2 \alpha\rangle}\left[\mathbb{F}_{\langle\alpha\rangle}\right]$. Irreducible, i.e., completely symmetric and traceless, tensors are designated by a prime, e.g., $\mathbb{V}_{\langle\alpha\rangle}^{\prime}$. The number of independent components of a tensor $\mathbb{V}_{\langle\alpha\rangle}$ is denoted by $\operatorname{dim}\left(\mathbb{V}_{\langle\alpha\rangle}\right)$. If it is necessary to distinguish tensors of the same rank, which are denoted by the same capital letter, then an additional index in the bracket specifying the tensor rank is introduced. For example $\mathbb{V}_{\left\langle 11_{1}\right\rangle}^{\prime}$ and $\mathbb{V}_{\left\langle 12_{2}\right\rangle}^{\prime}$ are two different 12th-order tensors. The set of proper orthogonal tensors is specified by $S O(3)$.

\section{Tensorial representation of the codf}

Properties of the codf. The orientation of a crystallite is described by a proper orthogonal tensor $\boldsymbol{Q}=\boldsymbol{g}_{i} \otimes \boldsymbol{e}_{i} \in S O(3)$ where $\boldsymbol{e}_{i}$ and $\boldsymbol{g}_{i}$ represent an orthonormal basis fixed to the sample and to the (orthonormal) lattice vectors of the crystal, respectively. The distribution of crystal orientations $\boldsymbol{Q}$ can be quantitatively described by the crystallite orientation distribution function $f(\boldsymbol{Q})[7,13]$. It represents the one-point correlation function of crystal orientations. For statistically homogeneous materials the one-point correlation function is position-independent. The function $f(\boldsymbol{Q})$ specifies the volume fraction of crystals having the orientation $Q$, i.e.,

$\frac{\mathrm{d} v}{v}(\boldsymbol{Q})=f(\boldsymbol{Q}) \mathrm{d} Q$.

$\mathrm{d} Q$ is the volume element in $S O(3)$, which ensures an invariant integration over $S O(3)$ [8]

$\int_{S O(3)} f(\boldsymbol{Q}) \mathrm{d} Q=\int_{S O(3)} f\left(\boldsymbol{Q} \boldsymbol{Q}_{0}\right) \mathrm{d} Q$

$\forall Q_{0} \in S O(3)$. If $S O(3)$ is parameterized by Euler angles $\left\{\varphi_{1}, \Phi, \varphi_{2}\right\}$, the volume element $\mathrm{d} Q$ is given by

$\mathrm{d} Q=\frac{\sin (\Phi)}{8 \pi^{2}} \mathrm{~d} \varphi_{1} \mathrm{~d} \Phi \mathrm{d} \varphi_{2}$.

The function $f(\boldsymbol{Q})$ is nonnegative and normalized such that 
$f(\boldsymbol{Q}) \geqslant 0 \forall \boldsymbol{Q} \in S O(3), \quad \int_{S O(3)} f(\boldsymbol{Q}) \mathrm{d} Q=1$.

In the present work, only time-independent textures are considered.

The orientation distribution function $f(\boldsymbol{Q})$ reflects both the symmetry of the crystallites forming the aggregate and the sample symmetry, which results from the processing history [22]. The crystal symmetry implies the following symmetry relation:

$f(\boldsymbol{Q})=f\left(\boldsymbol{Q} \boldsymbol{H}^{C}\right) \quad \forall \boldsymbol{H}^{C} \in S^{C} \subseteq S O(3)$,

where $S^{C}$ denotes the symmetry group of the crystallite. The sample symmetry implies

$f(\boldsymbol{Q})=f\left(\boldsymbol{H}^{S} \boldsymbol{Q}\right) \quad \forall \boldsymbol{H}^{S} \in S^{S} \subseteq S O(3)$.

$S^{S}$ denotes the symmetry group of the sample.

Tensorial Fourier expansion of the codf. For the subsequent considerations it is assumed that the codf is square integrable. This property implies the existence of a tensorial Fourier expansion. Adams et al. [1] and Guidi et al. [9] considered this expansion for the special case of a cubic crystal symmetry. Zheng and $\mathrm{Fu}[21,22]$ analyzed the expansion for arbitrary crystal and sample symmetries.

For aggregates of cubic crystals the Fourier expansion has the following form:

$f(\boldsymbol{Q})=1+\sum_{i=1}^{\infty} f_{\alpha_{i}}(\boldsymbol{Q})$,

where

$f_{\alpha_{i}}=\mathbb{V}_{\left\langle\alpha_{i}\right\rangle}^{\prime} \cdot \mathbb{F}_{\left\langle\alpha_{i}\right\rangle}^{\prime}(\boldsymbol{Q}), \quad \mathbb{F}_{\left\langle\alpha_{i}\right\rangle}^{\prime}(\boldsymbol{Q})=\boldsymbol{Q} \star \mathbb{T}_{\left\langle\alpha_{i}\right\rangle}^{\prime}$

and

$\left\{\alpha_{i}\right\}=\left\{4,6,8,9,10,12_{1}, 12_{2}, 13,14, \ldots\right\}$.

The $\mathbb{V}_{\left\langle\alpha_{i}\right\rangle}^{\prime}$ are called tensorial Fourier coefficients or texture coefficients. The tensors $\mathbb{T}_{\left\langle\alpha_{i}\right\rangle}^{\prime}$ are called reference tensors, which are normalized without loss of generality

$\left\|\mathbb{T}_{\left\langle\alpha_{i}\right\rangle}^{\prime}\right\|=1$.

The $\star$ in Eq. $(8)_{2}$ denotes the Rayleigh product, which for tensors $\mathbb{T}=T_{i j \ldots l} \boldsymbol{e}_{i} \otimes \boldsymbol{e}_{j} \otimes \ldots \otimes \boldsymbol{e}_{l}$ of arbitrary rank is defined by

$\boldsymbol{Q} \star \mathbb{T}=T_{i j \ldots l}\left(\boldsymbol{Q} \boldsymbol{e}_{i}\right) \otimes\left(\boldsymbol{Q} \boldsymbol{e}_{j}\right) \otimes \ldots \otimes\left(\boldsymbol{Q} \boldsymbol{e}_{l}\right)$.
The product $\boldsymbol{Q} \star \mathbb{T}_{\left\langle\alpha_{i}\right\rangle}^{\prime}$ can be interpreted as the rotation of the tensor $\mathbb{T}_{\left\langle\alpha_{i}\right\rangle}^{\prime}$ by the orthogonal tensor $\boldsymbol{Q}$.

The $\mathbb{V}_{\left\langle\alpha_{i}\right\rangle}^{\prime}$ and $\mathbb{T}_{\left\langle\alpha_{i}\right\rangle}^{\prime}$ are completely symmetric and traceless tensors. E.g., the following relations hold for $\mathbb{V}_{\langle 4\rangle}^{\prime}$

$V_{i j k l}^{\prime}=V_{j i k l}^{\prime}=V_{k l i j}^{\prime}=V_{k j l}^{\prime}=\ldots, V_{i k k l}^{\prime}=0$.

Completely symmetric and traceless tensors are called irreducible. An irreducible tensor $\mathbb{V}_{\left\langle\alpha_{i}\right\rangle}^{\prime}$ has $\operatorname{dim}\left(\mathbb{V}_{\left\langle\alpha_{i}\right\rangle}^{\prime}\right)=2 \alpha_{i}+1$ independent components. Hence, the number of independent components increases linearly with the tensor rank.

From Eqs. (5)-(8) it can be concluded that the reference tensors $\mathbb{T}_{\left\langle\alpha_{i}\right\rangle}^{\prime}$ reflect the crystal symmetry $f(\boldsymbol{Q})=f\left(\boldsymbol{Q} \boldsymbol{H}^{C}\right) \Rightarrow \mathbb{T}_{\left\langle\alpha_{i}\right\rangle}^{\prime}=\boldsymbol{H}^{C} \star \mathbb{T}_{\left\langle\alpha_{i}\right\rangle}^{\prime}$

$\forall \boldsymbol{H}^{C} \in S^{C}$, whereas the tensorial Fourier coefficients $\mathbb{V}_{\left\langle\alpha_{i}\right\rangle}^{\prime}$ have the sample symmetry

$f(\boldsymbol{Q})=f\left(\boldsymbol{H}^{S} \boldsymbol{Q}\right) \Rightarrow \mathbb{V}_{\left\langle\alpha_{i}\right\rangle}^{\prime}=\boldsymbol{H}^{S} \star \mathbb{V}_{\left\langle\alpha_{i}\right\rangle}^{\prime}$

$\forall \boldsymbol{H}^{S} \in S^{S}$.

The Fourier coefficients $\mathbb{V}_{\left\langle\alpha_{i}\right\rangle}^{\prime}$ can be considered as micro-mechanically based tensorial internal variables. They are defined in terms of the one-point correlation function of crystal orientations, which can be determined by texture measurements. Bunge [7] and Roe [13] used generalized harmonic functions in their representation of the codf, the coefficients of which are not tensor components.

It can be shown that the Voigt bound and the Reuss bound of the strain energy of linear elastic materials depend only on the fourth-order tensorial coefficient $\mathbb{V}_{\langle 4\rangle}^{\prime}$ [5]. It has also been shown how a quadratic yield function of weakly textured fcc polycrystals can be specified in terms of the texture coefficient $\mathbb{V}_{\langle 4\rangle}^{\prime}[5]$.

Calculation of the Fourier coefficients. In the context of a tensorial Fourier expansion of the codf, the orthogonality relations are given by

$\int_{S O(3)} \mathbb{F}_{\left\langle\alpha_{i}\right\rangle}^{\prime}(\boldsymbol{Q}) \otimes \mathbb{F}_{\left\langle\alpha_{j}\right\rangle}^{\prime}(\boldsymbol{Q}) \mathrm{d} Q=\mathbb{O} \quad \forall i \neq j$

and

$\int_{S O(3)} \mathbb{F}_{\left\langle\alpha_{i}\right\rangle}^{\prime}(\boldsymbol{Q}) \otimes \mathbb{F}_{\left\langle\alpha_{i}\right\rangle}^{\prime}(\boldsymbol{Q}) \mathrm{d} Q=\frac{1}{2 \alpha_{i}+1} \boldsymbol{\Delta}_{\left\langle 2 \alpha_{i}\right\rangle}$. 
$\boldsymbol{\Delta}_{\left\langle 2 \alpha_{i}\right\rangle}$ denotes the identity on irreducible tensors of rank $\alpha_{i}$. A combination of equations (7) and (15) gives the following set of linear equations:

$\mathbb{J}_{\left\langle\alpha_{i}\right\rangle}^{\prime}=\mathbb{E}_{\left\langle 2 \alpha_{i}\right\rangle}\left[\mathbb{V}_{\left\langle\alpha_{i}\right\rangle}^{\prime}\right]$

where

$\mathbb{J}_{\left\langle\alpha_{i}\right\rangle}^{\prime}=\int_{S O(3)} f(\boldsymbol{Q}) \mathbb{F}_{\left\langle\alpha_{i}\right\rangle}^{\prime}(\boldsymbol{Q}) \mathrm{d} Q$

and

$\mathbb{E}_{\left\langle 2 \alpha_{i}\right\rangle}=\int_{S O(3)} \mathbb{F}_{\left\langle\alpha_{i}\right\rangle}^{\prime}(\boldsymbol{Q}) \otimes \mathbb{F}_{\left\langle\alpha_{i}\right\rangle}^{\prime}(\boldsymbol{Q}) \mathrm{d} Q$

Eq. (16) can now be used to simplify (19) and to solve the system of equations. Finally one obtains

$\mathbb{V}_{\left\langle\alpha_{i}\right\rangle}^{\prime}=\left(2 \alpha_{i}+1\right) \int_{S O(3)} f(\boldsymbol{Q}) \mathbb{F}_{\left\langle\alpha_{i}\right\rangle}^{\prime}(\boldsymbol{Q}) \mathrm{d} Q$.

For a set of discrete crystal orientations and corresponding volume fractions $\left\{\boldsymbol{Q}_{\beta}, v_{\beta}\right\}$ $(\beta=1, \ldots, N)$ the codf can be approximated by

$f \approx \sum_{\beta=1}^{N} v_{\beta} \delta_{\boldsymbol{Q}_{\beta}}$.

Eqs. (20) and (21) imply the following formula for the texture coefficients:

$\mathbb{V}_{\left\langle\alpha_{i}\right\rangle}^{\prime}=\left(2 \alpha_{i}+1\right) \sum_{\beta=1}^{N} v_{\beta} \boldsymbol{Q}_{\beta} \star \mathbb{T}_{\left\langle\alpha_{i}\right\rangle}^{\prime}$.

\section{The maximum entropy method}

Texture entropy. In establishing information theory, Shannon [15] found a unique quantity that measured the uncertainty of an information source. Due to the similarity of the above mentioned quantity and the entropy used in thermodynamics, it was called entropy. In order to distinguish both quantities it is also called information-theoretic entropy or Shannon's entropy.

The maximum entropy method or maximum entropy principle was introduced by Jaynes $[10,11]$ into the field of statistical mechanics. For an overview on this method see the book by $\mathrm{Wu}$ [20]. For an application of the information-theoretic entropy in the context of orientational statistics see Mardia and Jupp [12] and Schaeben [14].
The information-theoretic entropy of the orientation distribution function is defined by

$E=-\int_{S O(3)} f(\boldsymbol{Q}) \ln (f(\boldsymbol{Q})) \mathrm{d} Q \in(-\infty, 0]$.

It is equal to zero for a uniform distribution and smaller than zero otherwise.

Formulation of the moment problem. Consider the following problem: how to determine an estimate $\bar{f}(\boldsymbol{Q})$ of the function $f(\boldsymbol{Q})$, if $L$ tensorial Fourier coefficients $\mathbb{V}_{\left\langle\alpha_{i}\right\rangle}^{\prime}(i=1, \ldots, L)$ are known? The estimate has to be consistent in the sense that (4) and $(4)_{2}$ are fulfilled.

For a given set of discrete crystal orientations and corresponding volume fractions, the Fourier coefficients can be estimated by Eq. (22) up to an arbitrary tensor rank. Therefore, the moment problem can also be stated in this case.

It should be noted that a truncation of the series (7) would generally give an inconsistent estimate of the codf, because the constraint (4) $)_{1}$ can be violated

$f(\boldsymbol{Q})=1+\sum_{i=1}^{L} \mathbb{V}_{\left\langle\alpha_{i}\right\rangle}^{\prime} \cdot \mathbb{F}_{\left\langle\alpha_{i}\right\rangle}^{\prime}(\boldsymbol{Q}) \ngtr 0$

if $L$ is finite. This will be shown later for specific textures. In the context of the representation of the codf by generalized harmonics this problem has been considered by [18].

The moment problem is well known in statistical mechanics and other areas of science. The problem of estimating a distribution function based on incomplete data has no unique solution and therefore it is ill-posed. One way to single out a solution of the aforementioned problem is to maximize the entropy of the estimate $\bar{f}(\boldsymbol{Q})$ of $f(\boldsymbol{Q})$

$\bar{E}=-\int_{S O(3)} \bar{f}(\boldsymbol{Q}) \ln (\bar{f}(\boldsymbol{Q})) \mathrm{d} Q \rightarrow \max$.

This is a nonlinear constrained optimization problem. The first constraint is given by the normalization condition $(4)_{1}$, which implies

$G_{0}:=\int_{S O(3)} \bar{f}(\boldsymbol{Q}) \mathrm{d} Q-1=0$.

The other constraints are given by the requirement that the $L$ leading Fourier coefficients of $\bar{f}(\boldsymbol{Q})$ are 
equal to the given coefficients of the distribution function $f(\boldsymbol{Q})$

$\mathbb{G}_{\alpha_{i}}:=\left(2 \alpha_{i}+1\right) \int_{S O(3)} \bar{f}(\boldsymbol{Q}) \mathbb{F}_{\left\langle\alpha_{i}\right\rangle}^{\prime}(\boldsymbol{Q}) \mathrm{d} Q-\mathbb{V}_{\left\langle\alpha_{i}\right\rangle}^{\prime}=\mathbb{O}$,

where $i=1, \ldots, L$.

As mentioned before, a Fourier coefficient $\mathbb{V}_{\left\langle\alpha_{i}\right\rangle}^{\prime}$ has $2 \alpha_{i}+1$ independent components. Hence, for $L$ given coefficients, (26) and (27) represent

$1+\sum_{i=1}^{L}\left(2 \alpha_{i}+1\right)$

independent nonlinear equations.

Formal solution of the moment problem. The main result of this paper is the application of the maximum entropy method in the context of tensorial representation of the codf. The equations, which have to be solved for the estimate of the distribution function, are derived for all tensor ranks of the Fourier coefficients.

The constrained optimization problem (25) and (26) can be solved by the Lagrange multiplier method. The objective functional $\mathscr{L}$ is

$\mathscr{L}=\bar{E}-\bar{V}_{0}^{*} G_{0}-\sum_{i=1}^{L} \overline{\mathbb{V}}_{\left\langle\alpha_{i}\right\rangle}^{\prime} \cdot \mathbb{G}_{\alpha_{i}}$

where $V_{0}^{*}$ and $\overline{\mathbb{V}}_{\left\langle\alpha_{i}\right\rangle}^{\prime}$ are the Lagrange multipliers. The first variation of $\mathscr{L}$ with respect to $\bar{f}$ is

$\delta \mathscr{L}=\int_{S O(3)} h(\boldsymbol{Q}) \delta \bar{f} \mathrm{~d} Q$

where

$h(\boldsymbol{Q})=\bar{V}_{0}+\ln (\bar{f}(\boldsymbol{Q}))+\sum_{i=1}^{L}\left(2 \alpha_{i}+1\right) \overline{\mathbb{V}}_{\left\langle\alpha_{i}\right\rangle}^{\prime} \cdot \mathbb{F}_{\left\langle\alpha_{i}\right\rangle}^{\prime}(\boldsymbol{Q})$

with $\bar{V}_{0}=1+\bar{V}_{0}^{*}$. After setting the first variation equal to zero and taking into account that $\delta \bar{f}$ is arbitrary, one gets the final form of the estimate of the codf

$\bar{f}(\boldsymbol{Q})=\mathrm{e}\left(-\bar{V}_{0}-\sum_{i=1}^{L}\left(2 \alpha_{i}+1\right) \overline{\mathbb{V}}_{\left\langle\alpha_{i}\right\rangle}^{\prime} \cdot \mathbb{F}_{\left\langle\alpha_{i}\right\rangle}^{\prime}(\boldsymbol{Q})\right)$
The estimate $\bar{f}(\boldsymbol{Q})$ is an exponential form in terms of the Lagrange multipliers, which have to be calculated by the constraints (26) and (27).

An exponential form similar to (31), but defined in terms of generalized harmonic functions, has been considered by van Houtte [19] and Van den Boogaart [16,17]. Van den Boogaart calls it crystallographic exponential family.

Numerical solution of the moment problem. The nonlinear constrained optimization problem given by (25)-(27) has been transformed into the set of $1+\sum_{i=1}^{L}\left(2 \alpha_{i}+1\right)$ nonlinear equations

$$
1=\int_{S O(3)} \bar{f}(\boldsymbol{Q}) \mathrm{d} Q
$$

and

$\mathbb{V}_{\left\langle\alpha_{i}\right\rangle}^{\prime}=\left(2 \alpha_{i}+1\right) \int_{S O(3)} \bar{f}(\boldsymbol{Q}) \mathbb{F}_{\left\langle\alpha_{i}\right\rangle}^{\prime}(\boldsymbol{Q}) \mathrm{d} Q$

$(i=1, \ldots, L)$, where

$\bar{f}(\boldsymbol{Q})=\mathrm{e}\left(-\bar{V}_{0}-\sum_{i=1}^{L}\left(2 \alpha_{i}+1\right) \overline{\mathbb{V}}_{\left\langle\alpha_{i}\right\rangle}^{\prime} \cdot \mathbb{F}_{\left\langle\alpha_{i}\right\rangle}^{\prime}(\boldsymbol{Q})\right)$.

If only $\mathbb{V}_{\langle 4\rangle}^{\prime}$ is known, then $L=1$ and the codf is estimated based on nine texture coefficients, i.e., the nine independent components of $\mathbb{V}_{\langle 4\rangle}^{\prime}$. In this case, the estimate (31) depends on the 10 parameters $\left\{\bar{V}_{0}, \mathbb{V}_{\langle 4\rangle}^{\prime}\right\}$ which are determined by the 10 constraints (32) and (33).

If $\mathbb{V}_{\langle 4\rangle}^{\prime}$ and $\mathbb{V}_{\langle 6\rangle}^{\prime}$ are known, then $L=2$ and the codf is estimated based on 22 texture coefficients, i.e., the nine independent components of $\mathbb{V}_{\langle 4\rangle}^{\prime}$ and the 13 independent components of $\mathbb{V}_{\langle 6\rangle}^{\prime}$. In this case, the estimate (31) depends on the 23 parameters $\left\{\bar{V}_{0}, \mathbb{V}_{\langle 4\rangle}^{\prime}, \mathbb{V}_{\langle 6\rangle}^{\prime}\right\}$ which are determined by the 23 constraints (32) and (33).

The numerical solution of (32) and (33) is obtained by a damped form of Newton's method. The Jacobian of the system of nonlinear equations have been numerically determined. The numerical solution of these equations requires a numerical integration over $S O(3)$, which has been performed by an adaptive integration scheme $[2,3]$. 


\section{Numerical example}

Fig. 1 shows a model codf due to a tensile deformation with an equivalent strain of 0.5 as it is predicted by full-constrained Taylor model. The Taylor simulation has been performed with 3000 equal-sized face-centered cubic single crystals. The codf has been estimated by a superposition of 3000 Mises-Fisher distributions [12], one for each crystal orientation, and their crystallographic equivalents due to the cubic crystal symmetry. The half-width has been taken to be equal to $12.5^{\circ}$.

Based on the single crystal orientations given by the Taylor simulation, the tensorial Fourier coefficients have been estimated by Eq. (22). In Figs. 2 and 3, inconsistent estimates of the codf based on a truncation of the tensorial Fourier expansion are shown. The inconsistency is obvious because the truncation leads to negative values of the codf.

In Figs. 4-6 the solution of the moment problem can bee seen for the cases that $\left\{\mathbb{V}_{\langle 4\rangle}^{\prime}\right\}$, $\left\{\mathbb{V}_{\langle 4\rangle}^{\prime}, \mathbb{V}_{\langle 6\rangle}^{\prime}\right\}$ and $\left\{\mathbb{V}_{\langle 4\rangle}^{\prime}, \mathbb{V}_{\langle 6\rangle}^{\prime}, \mathbb{V}_{\langle 8\rangle}^{\prime}\right\}$ are taken into
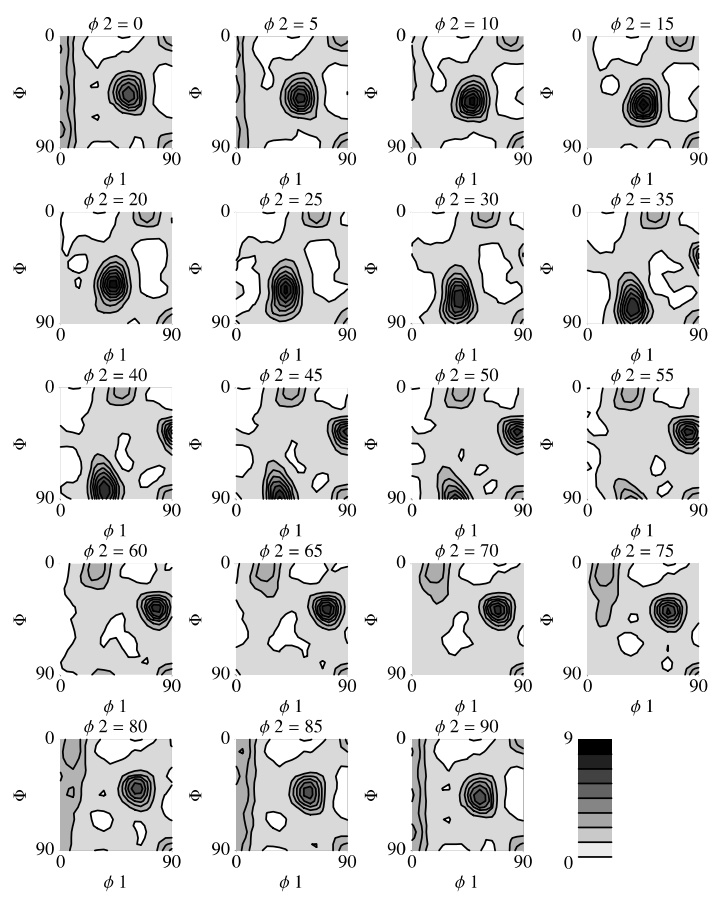

Fig. 1. Estimate of the codf by a superposition of Mises-Fisher distributions (tensile test, equivalent strain 0.5 ).
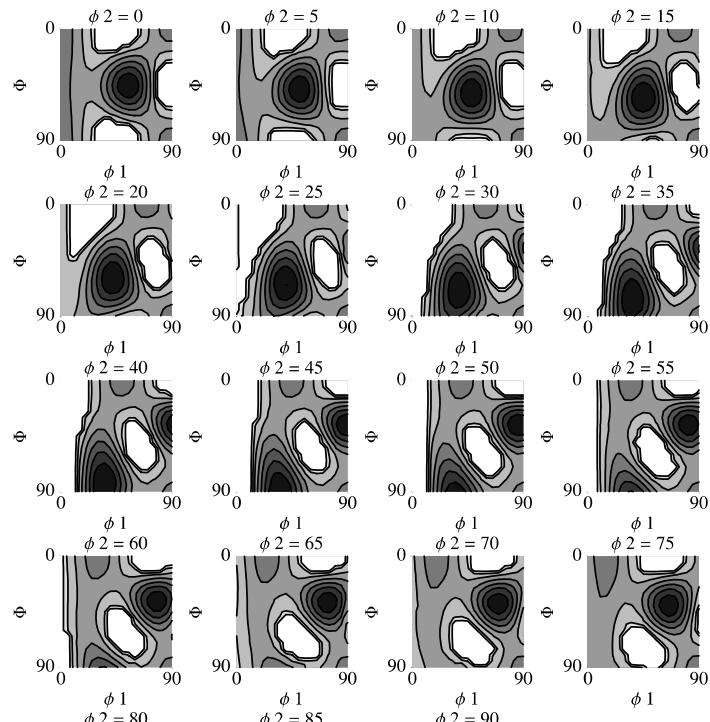

$\phi 1$
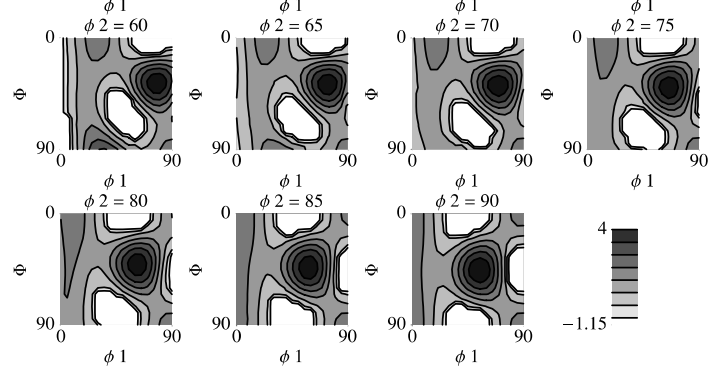

Fig. 2. Approximation of the codf based on $\mathbb{V}_{\langle 4\rangle}^{\prime}$ and $\mathbb{V}_{\langle 6\rangle}^{\prime}$ : $\bar{f}(\boldsymbol{Q}) \approx 1+\mathbb{V}_{\langle 4\rangle}^{\prime} \cdot \mathbb{F}_{\langle 4\rangle}^{\prime}(\boldsymbol{Q})+\mathbb{V}_{\langle 6\rangle}^{\prime} \cdot \mathbb{F}_{\langle 6\rangle}^{\prime}(\boldsymbol{Q})$.
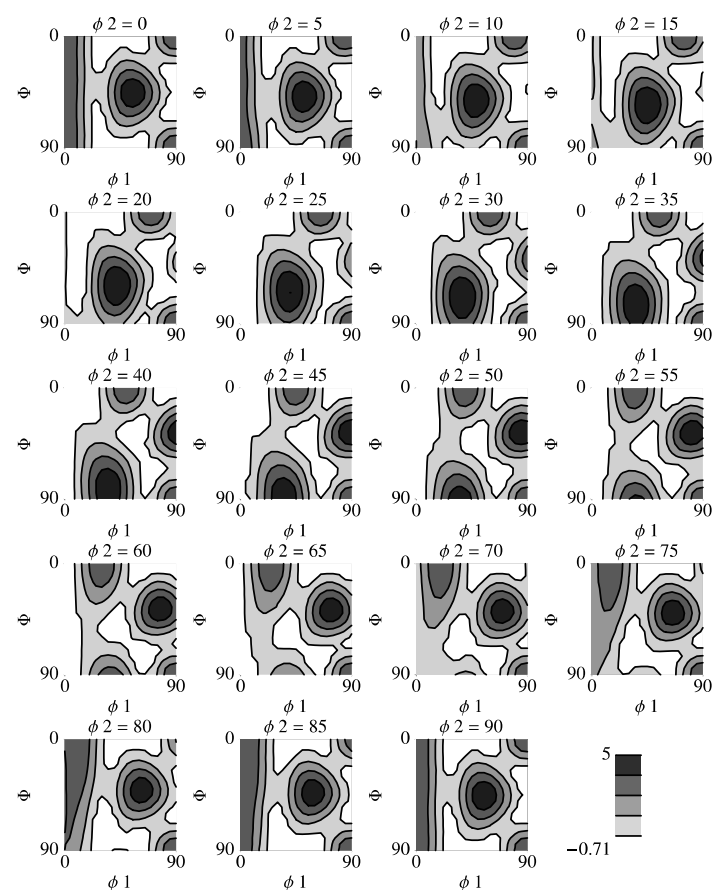

$\phi 1$

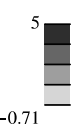

Fig. 3. Approximation of the codf based on $\mathbb{V}_{\langle 4\rangle}^{\prime}, \mathbb{V}_{\langle 6\rangle}^{\prime}$, and $\mathbb{V}_{\langle 8\rangle}^{\prime}: \bar{f}(\boldsymbol{Q}) \approx 1+\mathbb{V}_{\langle 4\rangle}^{\prime} \cdot \mathbb{F}_{\langle 4\rangle}^{\prime}(\boldsymbol{Q})+\cdots+\mathbb{V}_{\langle 8\rangle}^{\prime} \cdot \mathbb{F}_{\langle 8\rangle}^{\prime}(\boldsymbol{Q})$. 

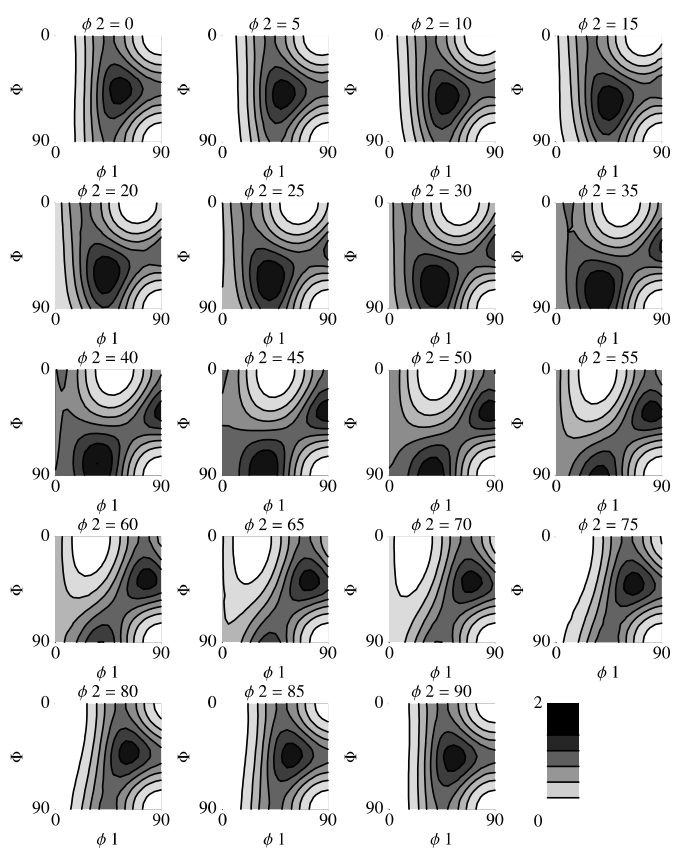

Fig. 4. Maximum entropy estimate of the codf based on $\mathbb{V}_{\langle 4}^{\prime}$ (nine texture coefficients): $\bar{f}(\boldsymbol{Q})=\exp \left(\bar{V}_{0}-9 \overline{\mathbb{V}}_{\langle 4\rangle}^{\prime} \cdot \mathbb{F}_{\langle 4\rangle}^{\prime}(\boldsymbol{Q})\right.$ ).
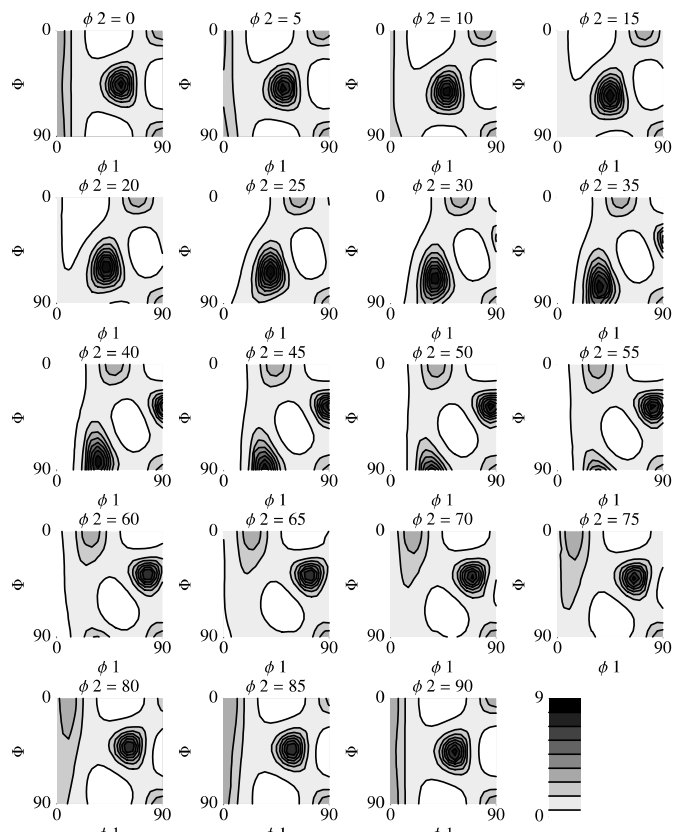

$\phi 1$

Fig. 5. Maximum entropy estimate of the codf based on $\mathbb{V}^{\prime}{ }^{\prime 4\rangle}$ and $\mathbb{V}_{\langle 6\rangle}^{\prime}(22$ texture coefficients $): \bar{f}(\boldsymbol{Q})=\exp \left(\bar{V}_{0}-9 \overline{\mathbb{V}}_{\langle 4\rangle}^{\prime 4\rangle}\right.$. $\left.\mathbb{F}_{\langle 4\rangle}^{\prime}(\boldsymbol{Q})-13 \overline{\mathbb{V}}_{\langle 6\rangle}^{\prime} \cdot \mathbb{F}_{\langle 6\rangle}^{\prime}(\boldsymbol{Q})\right)$.

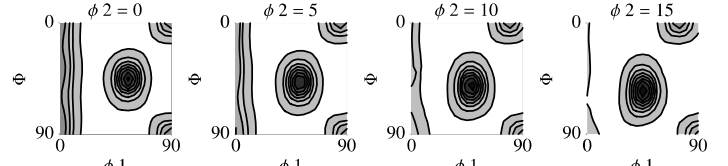

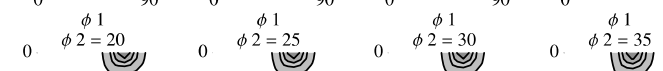
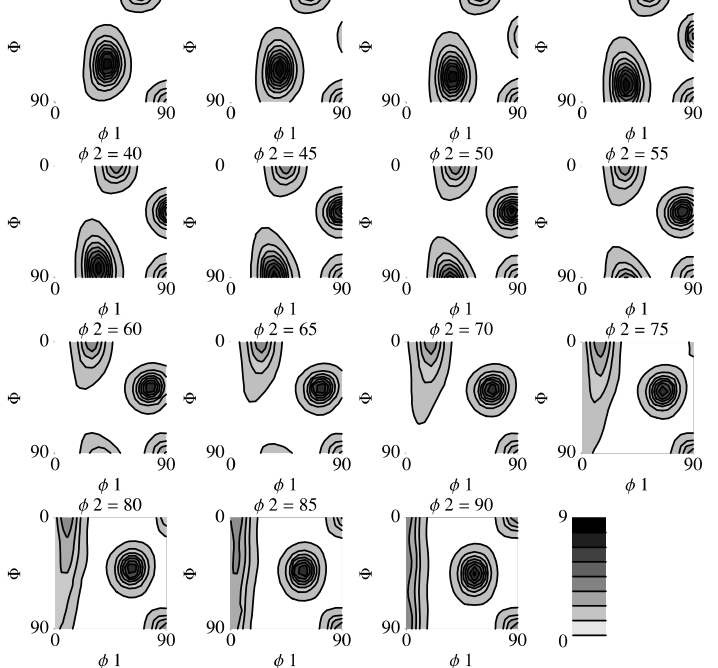

$\phi 1$

Fig. 6. Maximum entropy estimate of the codf based on $\mathbb{V}^{\prime}{ }_{4\rangle}$, $\mathbb{V}_{\langle 6\rangle}^{\prime}$, and $\mathbb{V}_{\langle 8\rangle}^{\prime}$ (39 texture coefficients): $\bar{f}(\boldsymbol{Q})=\exp \left(\bar{V}_{0}-9 \overline{\mathbb{V}}_{\langle 4\rangle}^{\prime 4\rangle}\right.$. $\left.\mathbb{F}_{\langle 4\rangle}^{\prime}(\boldsymbol{Q})-\cdots-17 \overline{\mathbb{V}}_{\langle 8\rangle}^{\prime} \cdot \mathbb{F}_{\langle 8\rangle}^{\prime}(\boldsymbol{Q})\right)$.

account. The maximum entropy estimate based only on $\mathbb{V}_{\langle 4\rangle}^{\prime}$ is not acceptable because important parts of the texture are missing. The main features of this tensile texture are reproduced by the estimate based on $\left\{\mathbb{V}_{\langle 4\rangle}^{\prime}, \mathbb{V}_{\langle 6\rangle}^{\prime}\right\}$.

\section{Summary}

It has been shown how the maximum entropy method can be applied to estimate the crystallite orientation distribution function, which is defined on the special orthogonal group. Special emphasis is given to a coordinate-free representation of the distribution function. The moment problem, which occurs in that context, has been derived and formally solved based on the entropy maximization. As a result, the estimate of the codf has an exponential form. The tensor-valued functions, which have to be solved in order to specify the exponential form, have been formulated for all tensor ranks that occur in the tensorial Fourier expansion. As a numerical example, the orientation distribution 
due to a tensile deformation has been estimated based on the maximum entropy method.

\section{References}

[1] B. Adams, J. Boehler, M. Guidi, E. Onat, Group theory and representation of microstructure and mechanical behavior of polycrystals, J. Mech. Phys. Solids 40 (4) (1992) 723-737.

[2] J. Berntsen, T. Espelid, An adaptive algorithm for the approximate calculation of multiple integrals, ACM Trans. Math. Software 17 (4) (1991) 437-451.

[3] J. Berntsen, T. Espelid, Algorithm 698: DCUHRE: an adaptive multidimensional integration routine for a vector of integrals, ACM Trans. Math. Software 17 (4) (1991) 452-456.

[4] T. Böhlke, A. Bertram, The evolution of Hooke's law due to texture development in polycrystals, Int. J. Solids Struct. 38 (52) (2001) 9437-9459.

[5] T. Böhlke, A. Bertram, Crystallographic texture induced anisotropy in copper: an approach based on a tensorial Fourier expansion of the codf, J. Phys. IV 105 (2003) 167174.

[6] T. Böhlke, A. Bertram, Modeling of deformation induced anisotropy in free-end torsion, Int. J. Plast. 19 (2003) 18671884.

[7] H.-J. Bunge, Zur Darstellung allgemeiner Texturen, Z. Metallkde 56 (1965) 872-874.

[8] I. Gel'fand, R. Minlos, Z. Shapiro, Representations of the Rotation and Lorentz Groups and their Applications, Pergamon Press, Oxford, 1963.

[9] M. Guidi, B. Adams, E. Onat, Tensorial representation of the orientation distribution function in cubic polycrystals, Textures Microstruct. 19 (1992) 147-167.

[10] E. Jaynes, Information theory and statistical mechanics, Phys. Rev. 106 (1957) 620-630.
[11] E. Jaynes, Information theory and statistical mechanics ii, Phys. Rev. 108 (1957) 171-190.

[12] K. Mardia, P. Jupp, Directional Statistics, John Wiley and Sons Ltd., Chichester, 2000.

[13] R. Roe, Description of crystalline orientation of polycrystalline material. III. General solution to pole figure inversion, J. Appl. Phys. 36 (1965) 2024-2031.

[14] H. Schaeben, Diskrete mathematische Methoden zur Berechnung und Interpretation von kristallographischen Orientierungsdichten, DGM Informationsgesellschaft $\mathrm{mbH}, 1994$.

[15] C. Shannon, A mathematical theory of communication, Bell Syst. Tech. J. 27 (379-423) (1948) 623-656.

[16] K. van den Boogaart, Statistics for individual orientation measurements, In: Proceedings of the 12th International Conference on Textures of Materials (ICOTOM-12), 1999, pp. 162-167.

[17] K. van den Boogaart, Statistics for Individual Crystallographic Orientation Measurements, Shaaker Verlag, Aachen, 2002.

[18] P. van Houtte, The use of a quadratic form for the determination of nonnegative texture functions, Texture Microstruct. 6 (1983) 1-20.

[19] P. van Houtte, A method for the generation of various ghost correction algorithms - the example of the positivity method and the exponential method, Texture Microstruct. 13 (1991) 199-212.

[20] N. Wu, The Maximum Entropy Method. Springer Series in Information Sciences, Springer, Berlin, 1997.

[21] Q.-S. Zheng, Y.-B. Fu, Orientation distribution functions for microstructures of heterogeneous materials: I directional distribution functions and irreducible tensors, Appl. Math. Mech. 22 (8) (2001) 865-884.

[22] Q.-S. Zheng, Y.-B. Fu, Orientation distribution functions for microstructures of heterogeneous materials: II crystal distribution functions and irreducible tensors restricted by various material symmetries, Appl. Math. Mech. 22 (8) (2001) 885-903. 